\section{Knie-TEP: Das Für und Wider abwägen \\ Herz-Risiko auch bei Bechterew \& PsA}

Etwa 158.000-mal pro Jahr wird in Deutschland ein Kunstknie implantiert. Die Evidenz für den Nutzen dieser Maßnahme als Ultima Ratio bei fortgeschrittener Gonarthrose war bis dato mäßig - Jetzt liegt erstmals eine qualitativ hochwertige Vergleichsstudie vor. Verglichen wurden zwei Strategien: Die Op. mit Knie-TEP gefolgt von konservativen Maßnahmen, bzw. nichtchirurgische Maßnahmen allein. Bei Schmerzen, allgemeiner Symptomatik, Alltagsaktivitäten und Lebensqualität schnitt die TEP insgesamt signifikant besser ab. Entscheidender Nachteil des chirurgischen Eingriffs: die deutlich höhere Rate schwerer Nebenwirkungen.
$\mathrm{Zu}$ den klinisch relevanten Aspekten in der Betreuung von Patienten mit Spondylarthropathien (SpA) sollte auch die regelmäßige Überprüfung kardiovaskulärer Risiken gehören - so das Fazit mehrerer Untersuchungen beim ACR 2015. Denn die Hazard Ratio für ein akutes Koronarsyndrom betrug bei einer Psoriasis-Arthritis (PsA) 1,71 und 1,41 für einen Schlaganfall, bei Ankylosierender Spondylitis (AS) 1,47 bzw. 1,33 gegenüber der gesunden Bevölkerungskohorte. Venöse thromboembolische Ereignisse sind um 50\% häufiger; auch Vorhofflimmern, AV-Block und Schrittmacherimplantationen waren bei SpA-Patienten etwa doppelt so häufig.

Abstract 1057/1058/1059, 2015 ACR/ARHP, San Francisco

\section{Alle Jahre wieder...}

Weihnachten: Familien finden zueinander, es duftet nach Plätzchen und Tannengrün und andererseits werden wieder fleißig Patienten generiert: Vorweihnachtliche GlühweinExzesse und Feiertags-Völlereien lassen landesweit Harnsäurepegel steigen, Hobbyweihnachtsmänner werden zum Dank mit Rückenschmerzen beschert, die Pistengaudi endet jäh mit kaputtem Gelenk und allzu oft wird der "Gute Rutsch" wörtlich genommen. Oh, du Fröhliche!

Wappnen Sie sich mit neuem Wissen aus unserem Schwerpunkt "Knochen \& Gelenke".

Melanie Goldschmidt Redakteurin

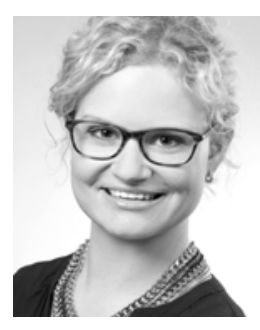

\title{
Chondroitinsulfat doch effektiv bei Kniegelenksarthrose
}

Ob Patienten mit aktivierter Kniegelenksarthrose mehr von Celecoxib oder Chondroitinsulfat profitieren, war Frage einer kanadischen Studie. Sie attestierte Chondroitinsulfat eine Überlegenheit. Das Team um Prof. Dr. Jean-Pierre Pelletier, Universität Montreal (Kanada), untersuchte die Progressionsverzögerung mittels quantitativer Magnetresonanztomografie (qMRT) bei 194 Gonarthrose-Patienten mit Synovitis. Je die Hälfte erhielt 1200 $\mathrm{mg} / \mathrm{d}$ einer pharmazeutischen Präparation von Chondroitinsulfat oder $200 \mathrm{mg} / \mathrm{d} \mathrm{Ce}$ lecoxib. Die Patienten durften zusätzlich Schmerzmittel einnehmen. Zu Beginn, nach einem und nach zwei Jahren wurde eine qMRT-Aufnahme gemacht, welche die gesamte Kniestruktur wiedergeben kann. „Der Knorpelverlust unterschied sich nach einem und zwei Jahren signifikant von dem in der Celecoxib-Gruppe“, so Pelletier.

Pelletier JP et al. Abstract 950. 2015 ACR/ARHP Annual Meeting, 7.-11. November, San Francisco

\section{GRAPPA-Empfehlung bei Psoriasis-Arthritis}

$\mathrm{Zu}$ den Late-breaking Abstracts beim ACR-Kongress gehörte auch die neue GRAPPA-Empfehlung zur Therapie der Psoriasis-Arthritis. Im Gegensatz zur EULAR-Empfehlung von diesem Sommer waren auch Dermatologen an der Aktualisierung beteiligt, bemerkte Professor Dr. Laura Coates. Daher liege der Schwerpunkt nicht auf der peripheren Arthritis, sondern umfasse alle Manifestationen - Arthritis, Spondylitis, Enthesitis, Daktylitis, Hautund Nagelbefall - gleichermaßen.

Gesundheitszustand vorab ermitteln Das letztendliche Ziel der Therapie ist eine möglichst geringe Krankheitsaktivität bzw. Remission in allen Domänen. Außerdem sollte der funktionelle Status optimiert, die Lebensqualität verbessert und struktureller Schaden verringert werden. Betont wird die Bedeutung eines umfassenden Assessments des Patienten unter Einbeziehung aller Krankheitsmanifestationen und Komorbiditäten. Das gewählte Medikament sollte möglichst viele Domänen abdecken, so Coates. Zu berücksichtigen seien zudem Krankheitsaktivität, Grad der Beeinträchtigung und Prognosefaktoren.

Coates LC et al. Abstract 7L. 2015 ACR/ARHP Annual Meeting, November 7-11, San Francisco 